\title{
Application of IoT in the Prevention of Carbon Monoxide Poisoning
}

\author{
Wei-Ling Hsu, ${ }^{1}$ Chih-Yuan Ho, ${ }^{2}$ Chiu-Kuo Liang, ${ }^{3}$ \\ Yan-Chyuan Shiau, ${ }^{4 *}$ Hung-Nien Hsieh, ${ }^{5}$ and Shu-Chen Lai ${ }^{6}$ \\ ${ }^{1}$ School of Urban and Environmental Science, Huaiyin Normal University, \\ No. 111, ChangJang West Road, Huai'an City, Jiangsu Province 223300, China \\ ${ }^{2}$ Department of Civil Engineering, Chung Hua University, Hsinchu, \\ No. 707, WuFu Road, Section 2, Hsinchu 30012, Taiwan \\ ${ }^{3}$ Department of Computer Science and Information Engineering, Chung-Hua University, \\ No. 707, WuFu Road, Section 2, Hsinchu 30012, Taiwan \\ ${ }^{4}$ Department of Landscape Architecture, Chung Hua University, \\ No. 707, WuFu Road, Section 2, Hsinchu 30012, Taiwan \\ ${ }^{5}$ College of Architecture and Design, Chung Hua University, \\ No. 707, WuFu Road, Section 2, Hsinchu 30012, Taiwan \\ ${ }^{6}$ Department of Construction Management, Chung Hua University, \\ No. 707, WuFu Road, Section 2, Hsinchu 30012, Taiwan
}

(Received May 25, 2019; accepted October 8, 2019)

Keywords: carbon monoxide poisoning, Line-based notification system, Internet of Things (IoT), Arduino

Because carbon monoxide ( $\mathrm{CO}$ ) is a colorless and odorless gas, symptoms of $\mathrm{CO}$ poisoning are difficult to recognize, making $\mathrm{CO}$ a hidden killer in home environments. To prevent $\mathrm{CO}$ poisoning, we used artificial intelligence and Internet of Things (IoT) technology to develop a $\mathrm{CO}$ poisoning prevention system with the following functional features: (1) a CO sensor, which was installed in the bathroom at home and immediately activated to warn the household when the CO concentration was excessively high; (2) an electric window opener and a fan, which when activated performed forced ventilation and thereby reduced CO concentration; (3) a device that cut off the gas supply; (4) a Line application that notified family members and signaled the community management center to conduct emergency rescue operations; and (5) a mechanism for unlocking the door to allow people to enter for emergency rescue. The obtained results may help reduce the number of $\mathrm{CO}$ poisoning cases and casualties in the winter in Taiwan.

\section{Introduction}

\subsection{Background}

Carbon monoxide (CO) poisoning is a significant cause of illness and death in the United States (US). The internal combustion engine and stoves burning fossil fuels result in most cases of CO poisoning. ${ }^{(1)}$ According to the statistics of the Fire Department of the Ministry of

*Corresponding author: e-mail: ycshiau@ms22.hinet.net https://doi.org/10.18494/SAM.2019.2482 
the Interior, from January 1, 2018 to December 15, 2018, there were 54 cases of CO poisoning accidents in Taiwan, resulting in 11 deaths and 171 injuries. ${ }^{(2)}$ Because CO is a colorless and odorless gas, symptoms of $\mathrm{CO}$ poisoning are difficult to recognize, making $\mathrm{CO}$ a hidden killer in home environments. After inhaling excessive amounts of $\mathrm{CO}$, people generally experience mild discomfort such as fatigue and dizziness, eventually causing them to lose consciousness and die.

The amount of air required for gas combustion is approximately 25 to 31 times its volume. In an oxygen-rich environment, complete gas combustion produces nonhazardous carbon dioxide $\left(\mathrm{CO}_{2}\right)$, whereas incomplete gas combustion in an oxygen-deficient environment generates $\mathrm{CO}$. Compared with oxygen, $\mathrm{CO}$ forms bonds with hemoglobin in the blood that are 200-250 times stronger. Thus, $\mathrm{CO}$ replaces oxygen and binds to hemoglobin, forming $\mathrm{CO}$ hemoglobin $(\mathrm{COHb})$. This reduces the oxygen capacity of hemoglobin, causing insufficient oxygen in human body tissues and inducing the development of $\mathrm{CO}$ poisoning symptoms.

\subsection{Research motivation and objectives}

Internet of Things (IoT) sensor technology has become part of everyday applications, thus solving medical service difficulties in rural areas. In response to health care requirements, local governments have been using IoT and sensor technology in resident healthcare management. ${ }^{(3)}$ $\mathrm{CO}$ poisoning caused by natural gas water heaters is a common household accident. In a preliminary study conducted by Chen et al., a smart gateway Wi-Fi alarm system and IoT notifications on mobile devices reduced $\mathrm{CO}$ poisoning incidents. ${ }^{(4)}$ To prevent $\mathrm{CO}$ poisoning, in this study, we explored related problems and proposed the following solutions:

(1) Install a CO concentration sensor in the bathroom at home and connect it to a controller board. Next, install an alarm device, a window opening device, a fan, a gas shutoff device, an electric door lock, and a network connection.

(2) When the sensor detects an excessively high $\mathrm{CO}$ concentration, the system automatically activates the electric window opener and turns on the fan to perform forced ventilation, thereby reducing the $\mathrm{CO}$ concentration.

(3) The alarm is immediately activated to alert the tenant; meanwhile, the gas shutoff device is activated to cut off the gas supply.

(4) The prevention mechanism sends Line notification messages to inform the household and community management center while unlocking the door to allow rescuers to enter and perform emergency rescue operations.

\subsection{Problems to be solved}

With billions of smart sensors connected to IoT devices, a prevalent IoT application consisting of a smart home gateway has become the central point of connection for smart sensors that monitor home environments. ${ }^{(5,6)}$ The incorporation of IoT sensors has become increasingly extensive with advances in network technology. In this study, we propose a holistic CO poisoning prevention mechanism to solve the following problems. 
Because $\mathrm{CO}$ is an odorless gas, sensing high $\mathrm{CO}$ concentrations is difficult for residents who are indoors. A sensing component must be installed to measure $\mathrm{CO}$ concentration and send alerts via warning lights and sirens.

By the time $\mathrm{CO}$ reaches a high concentration, people who are inside might have already been poisoned, resulting in delayed reactions. Therefore, the prevention mechanism must automatically cut off the gas supply, automatically open the window, and automatically turn on the fan to allow forced ventilation, thereby reducing the $\mathrm{CO}$ concentration.

When CO poisoning occurs, people might have already lost consciousness, at which point the community management center or an emergency unit must be notified immediately. Considering that a locked door may hinder rescue attempts, the prevention mechanism must automatically unlock the door to allow rescuers to enter from outside and conduct emergency rescue.

\section{Literature Review}

\subsection{CO poisoning}

\subsubsection{Potential causes of $\mathrm{CO}$ poisoning}

$\mathrm{CO}$ is a toxic product produced by water heaters, burning charcoal, automobiles, natural gas, and the incomplete combustion of carbonyl compounds in household or industrial fuel burning processes. ${ }^{(7-9)}$ A breakdown of the causes is presented in Fig. $1 .^{(10)}$ Common CO poisoning scenarios are detailed as follows:

(1) Indoor gas water heaters can cause comas in individuals when they are bathing. Even if the gas water heater is installed on the balcony, the rooftop add-on causes dust to accumulate on window screens, resulting in insufficient air circulation, incomplete combustion, and consequently $\mathrm{CO}$ poisoning.

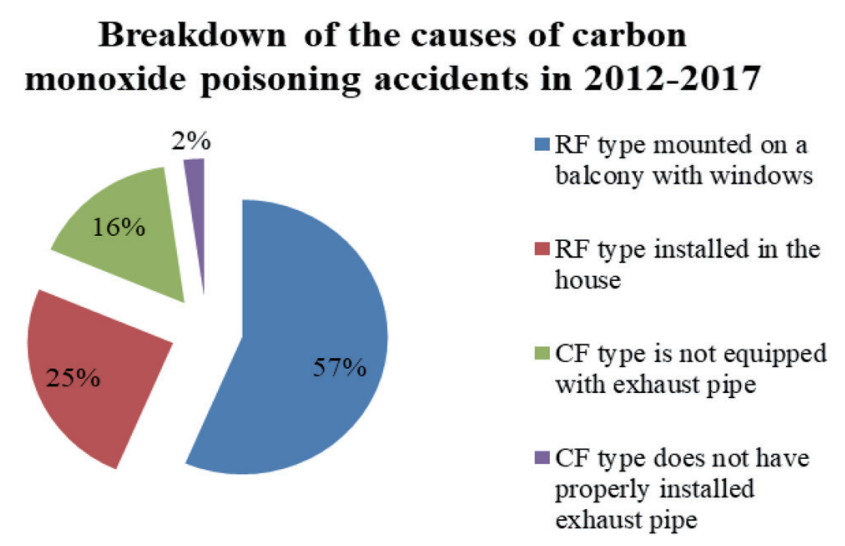

Fig. 1. (Color online) Pie chart of CO poisoning causes. RF denotes outdoor type, CF denotes conventional flue type, and FE denotes forced exhaust type. 
(2) Cooking hot pots or barbecuing with gas tanks or charcoal-burning cookware in an enclosed space on a cold day.

(3) Inhalation of exhaust from car engines in a poorly ventilated place such as a garage.

(4) Dense smoke generated from smoldering combustion before and after a major fire.

(5) Using a petroleum generator in an enclosed space during power failure.

(6) Individuals who turn on the faucet for a bath, forgetting to turn it off after discovering a water outage. Because commercial water heaters are generally water-ignited, when the water supply is restored, the water heater automatically ignites. At this point, insufficient indoor ventilation can lead to $\mathrm{CO}$ poisoning.

\subsubsection{Harmful effects of $\mathrm{CO}$ poisoning on the human body}

US Harvard Health Publications ${ }^{(11)}$ proposed the effects of $\mathrm{CO}$ content in relation to inhalation time and toxic symptoms, as shown in Table 1.

\subsubsection{Prevention of CO poisoning}

Because the initial symptoms of $\mathrm{CO}$ poisoning resemble those of a cold or food poisoning, such as exhaustion, upset stomach, and vomiting, they can easily be neglected, causing the tragedy of multiple people or entire families being simultaneously poisoned by $\mathrm{CO}$. The inhalation of $\mathrm{CO}$ hinders the bonding of oxygen to hemoglobin, resulting in organ hypoxia or damage to cell functions. Because people are often already weak by the time they realize their abnormal symptoms, seeking help may be difficult. To effectively prevent $\mathrm{CO}$ poisoning, the following five principles must be followed thoroughly: ${ }^{(12)}$

(1) Maintain sufficient ventilation in the house: avoid illegal use of balconies, the installation of add-on doors or windows, having unclean window screens, and hanging many clothes on the balcony.

(2) Purchase certified safe appliances: water heaters should be labeled with the Chinese National Standards Mark and Taiwan Gas Appliance Safety Mark.

Table 1

Physiological symptoms in relation to $\mathrm{CO}$ content and inhalation time.

\begin{tabular}{lc}
\hline CO content & Inhalation time and physiological symptoms \\
\hline $100 \mathrm{ppm}(0.01 \%)$ & Symptoms such as headache, dizziness, nausea, loss of muscle strength, and \\
impaired judgment within $6-8 \mathrm{~h}$ \\
$200 \mathrm{ppm}(0.02 \%)$ & Slight headache within $2-3 \mathrm{~h}$ \\
$400 \mathrm{ppm}(0.04 \%)$ & Intensified headache within $2.5-3.5 \mathrm{~h}$ \\
$800 \mathrm{ppm}(0.08 \%)$ & Dizziness, nausea, and convulsions within $45 \mathrm{~min}$ \\
$1600 \mathrm{ppm}(0.16 \%)$ & Headache and dizziness within 20 min; death within $2 \mathrm{~h}$ \\
$3200 \mathrm{ppm}(0.32 \%)$ & Headache, dizziness, and vomiting within 5-10 min; \\
$6400 \mathrm{ppm}(0.64 \%)$ & death within 30 min \\
$12800 \mathrm{ppm}(1.28 \%)$ & Headache and dizziness within $1-2$ min; death within $10-15 \mathrm{~min}$ \\
\hline
\end{tabular}


(3) Select the appropriate type of water heater: RF denotes outdoor type, CF denotes conventional flue type, FE denotes forced exhaust type, and FF denotes forced flue type.

(4) Ensure safe installation: appliances must be installed by qualified technicians in accordance with installation standards.

(5) Conduct routine overhaul: water heaters must be repaired or replaced regularly. Upon discovering unstable water temperatures or the need for a heater to be repositioned or a component to be replaced, a qualified technician must be called in for the implementation.

\subsection{IoT}

IoT is the carrier of information from the Internet and conventional telecommunications networks, and enables all common independently operating objects to interconnect with each other. IoT is generally a wireless network. Because the number of devices an individual comes into contact with can be as high as 1000-5000, IoT may include 500-1000 trillion objects. Each individual may connect a physical object to the Internet using an electronic tag, allowing the location of the object to be determined. Through the use of IoT, users can centrally manage and control machines, devices, and personnel using a central computer. Moreover, household appliances and automobiles can be remotely controlled; ${ }^{(13)}$ such a system resembles an automatic control system that allows objects to be tracked, thereby preventing theft. Data of these trivial items may be collected and integrated to form big data, which contribute to major social changes such as the redesign of roads to reduce the number of car accidents, urban renewal, disaster prediction, crime prevention, and epidemic control. IoT digitalizes the real world and has a wide range of applications. Because of its ability to integrate scattered information and organize digital information shared by objects, IoT is mainly applied in the fields of transportation and logistics, industrial manufacturing, medicine and health care, smart environments (e.g., homes, offices, and factories), and personal and social applications. Accordingly, IoT offers a broad range of marketing and application prospects. ${ }^{(14)}$

\subsection{Smart houses}

A smart house refers to the integration of all automation equipment through a network system to provide a service that is highly efficient overall, thereby ensuring that the public's needs are met in terms of home safety, a healthy and pleasant living environment, convenience, and quality of life. ${ }^{(15)}$ Residential buildings aim to provide a safe, convenient, healthy, and comfortable home environment, while meeting the needs of modern life as the primary concern. Therefore, the application of technological techniques for developing smart homes in accordance with life needs is an inevitable trend in current residential construction. ${ }^{(16)}$ Smart home designs mainly include the provision of life functions necessary for a house, such as safety, disaster prevention, health care, convenience, and comfort, and energy-saving and carbon-reducing functions for sustainable development. ${ }^{(17)}$ Realizing these functions requires a reliance on various devices for information communication and new services or technology. By creating an infrastructure platform, integration and proper management and maintenance can 
be performed to ensure tangible outcomes and enhance the quality of a living space, thereby achieving the objective of creating a smart house.

Smart houses embody different backgrounds and implications, and their definitions vary by generation, user needs, research topic, or industry and product characteristics. With the advent of the ubiquitous computing era in recent years, the construction as well as the information and communications technology industries are paying attention to the establishment of smart living space platforms by integrating electronics, information communication, and living spaces together. Moreover, the incorporation of the cloud computing industry has accelerated the development of residential smart service technology. Topics such as smart houses, smart offices, and smart cities have become the focus of industrial and academic research.

\subsection{Current state of research on $\mathrm{CO}$ poisoning prevention}

\subsubsection{Current CO poisoning prevention study}

$\mathrm{CO}$ is a stable oxide of carbon that is produced by the partial oxidation of carboncontaining compounds. ${ }^{(18)}$ In some reports, CO poisoning is responsible for more than half of accident-related deaths in many countries. Fatal cases of $\mathrm{CO}$ are also often misdiagnosed or underreported by professional medical personnel. Therefore, it is difficult to know the exact number of fatalities due to $\mathrm{CO}$ poisoning. Effects associated with $\mathrm{CO}$ poisoning include cardiovascular and neurobehavioral effects at low concentrations, as well as unconsciousness and death following exposure to higher concentrations of CO. Since CO poisoning is a frequently overlooked diagnosis, the measurement of $\mathrm{COHb}$ in the environment is particularly important. In addition, some early symptoms include headache, dizziness, weakness, nausea, confusion, and visual impairment. If these symptoms reoccur, special assessments are needed. Complications often occur in $\mathrm{CO}$ poisoning. Because myocardial tissue is very sensitive to the hypoxia of $\mathrm{CO}$, the heart is the most likely cause of immediate death from $\mathrm{CO}$ poisoning. Severe CO poisoning can cause significant hypotension, fatal arrhythmias, and ECG changes, and pulmonary edema may occur. The neurological manifestations of acute CO poisoning include disorientation, confusion, and coma. The most dangerous effect of $\mathrm{CO}$ poisoning is delayed neuromuscular injury and a slow regression of neurobehavioral consequences within 2-28 days after poisoning. CO poisoning during pregnancy usually causes fetal death, developmental disorders, and chronic brain damage leading to complications. In short, $\mathrm{CO}$ poisoning often occurs and has serious consequences, including immediate death, complications, and late sequelae, and is easily overlooked. The prevention of CO poisoning should be encouraged in public and medical education. ${ }^{(19)}$

\subsubsection{Current $\mathrm{CO}$ poisoning prevention patents}

We used the Global Patent Search System to search for related patented inventions, with the results detailed as follows: 


\subsubsection{Publication number: TW201317946A ${ }^{(20)}$}

As a home-based multihazard early warning system, this patent consists of a three-axis gravity acceleration sensor module, a smoke space detection module, a $\mathrm{CO}$ detection module, a microcontroller, and a wireless module, which enables the transmission of short messages and control over remote switches. The alarm device can send off a warning signal in the event of an earthquake, fire, or an excessively high $\mathrm{CO}$ concentration; when the three-axis gravity acceleration sensor as the above module detects an earthquake, the microcontroller activates an alarm to send a warning signal while turning off the gas switch and unlocking the front door through the wireless module. Comparing this system with the proposed system in this study, the aforementioned patent also has a CO detection module, an alarm device, a gas shutoff device, and a mechanism for unlocking the door; however, the patent lacks an automatic window opener, an automatic fan, and a Line-based notification system.

\subsubsection{Publication number: TWM552220U $\mathrm{U}^{(21)}$}

This patent is an IoT-based environment monitoring system composed of an environment monitoring device, an environment control device, a wireless terminal transmission device, and a terminal computer. The environment monitoring device was configured to acquire parameters for the environment control device to adjust the air quality. Comparing this system with the proposed system in this study, this patent employs an environment monitoring system in which the environment control device has an automatic window opener and an automatic fan but lacks a Line-based notification system.

\section{Research Design}

\subsection{Adopted software and hardware equipment}

The employed sensing components are detailed as follows.

\subsubsection{Arduino controller board}

Arduino $^{(22)}$ is a computer hardware and software company that designs and manufactures single-board microcontrollers and microcontroller kits for building digital devices and interactive objects that enable detection and control over objects in the physical and digital worlds. Arduino circuit boards employ various microprocessors and controllers. Some circuit boards are equipped with a series of digital and analog input/output pins that connect to various expansion boards, sensors, and other controllable components. These circuit boards have serial ports, such as universal serial bus ports, for loading programs onto personal computers. Microcontrollers often use $\mathrm{C} / \mathrm{C}++$ programming language. In addition to conventional compilation tool chains, Arduino projects offer an integrated development environment based on a processing language (Fig. 2). 


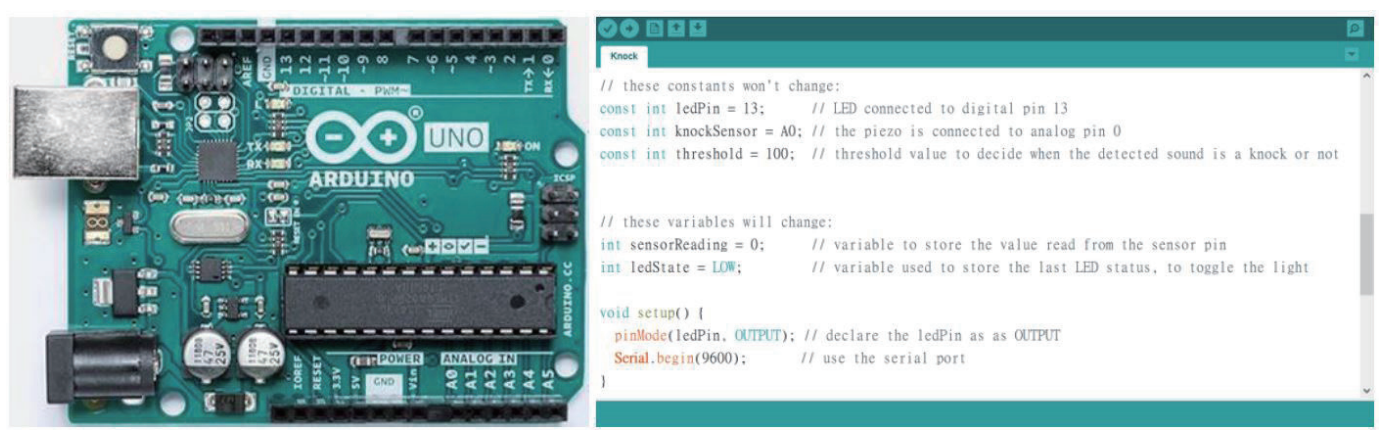

Fig. 2. (Color online) Arduino UNO controller board and a scene of the compiler.

\subsubsection{CO and gas sensor}

The CO sensor used in this study was the MQ-7 model (Fig. 3), ${ }^{(23)}$ which is used in CO detection devices in household environments for $\mathrm{CO}$ and gas detection. The MQ-7 gas sensor is highly sensitive to $\mathrm{CO}$. This type of sensor can detect a variety of CO-containing gases and is suitable for diverse applications.

\subsection{Proposed smart control mechanism}

After measuring the values of various indicators in the household environment through different sensors, a smart building uses the controller board and software to prompt relevant control mechanisms to send warning notifications and change physical indicator values, thereby creating a safe, comfortable, and pleasant home environment. The components and facilities used in this study to control the hardware environment and provide warnings are detailed as follows.

\subsubsection{Audible and visual alarm}

Upon detecting $\mathrm{CO}$, the audible and visual alarm (Fig. 4) sounds a siren and flashes strobe lights to notify residents to take relevant response measures.

\subsubsection{Electric window opener}

When the system detects a high $\mathrm{CO}$ concentration, it activates the electric window opener (Fig. 5) ${ }^{(24)}$ to open the windows for ventilation.

\subsubsection{Fan}

A fan was mounted to conduct forced ventilation for the rapid reduction in $\mathrm{CO}$ concentration, thereby mitigating the harmful effects of CO incurred by residents. An L9110 fan module was 


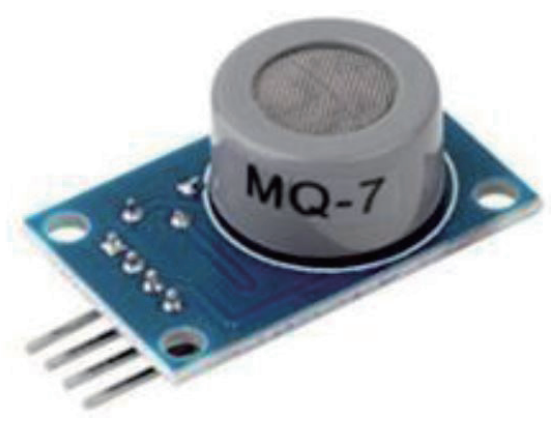

Fig. 3. (Color online) CO sensor.

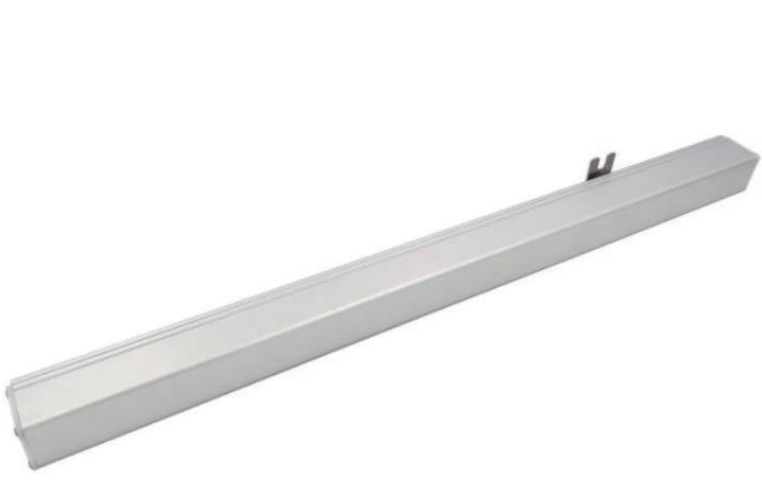

(a)

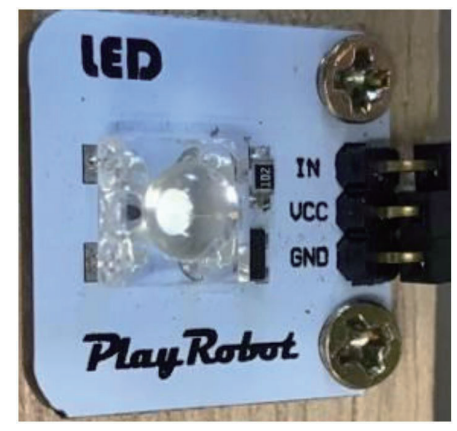

Fig. 4. (Color online) Audible and visual alarm.

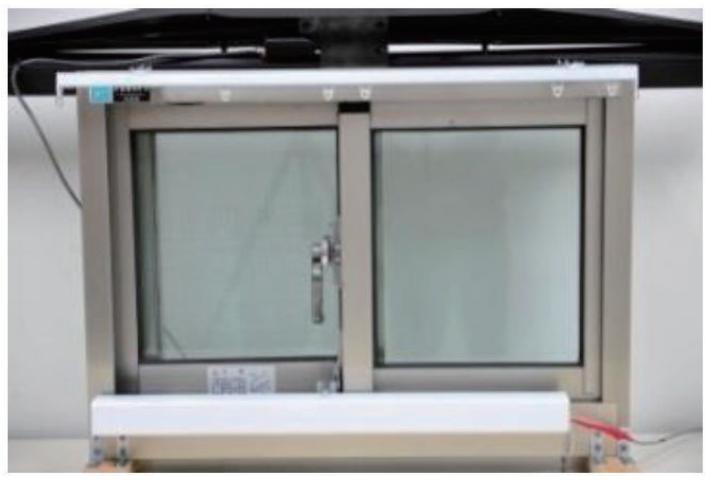

(b)

Fig. 5. (Color online) Electric window opener. (a) Electric window opener and (b) electric window opener mounted on window.

used in the simulation carried out in this study (Fig. 6), ${ }^{(25)}$ and a relay was connected to control the actual fan in the proposed mechanism.

\subsubsection{Gas shutoff device}

The detection of a high CO concentration immediately activated the gas shutoff device (Fig. 7), ${ }^{(26)}$ which rotated the switching valve $90^{\circ}$ clockwise to cut off the gas supply, thereby ensuring resident safety.

\subsubsection{IFTTT}

IFTTT $^{(27)}$ - if this, then that - is an automation platform that enables the connection of multiple Internet services. On the basis of the conditions of other platforms, IFTTT determines whether to execute a command. Triggered by task conditions, IFTTT resembles a programming language; that is, if XXX performs YYY behavior, then execute ZZZ. A "channel" is any website that fires a trigger or serves as a triggering event; "triggers" are the triggering 


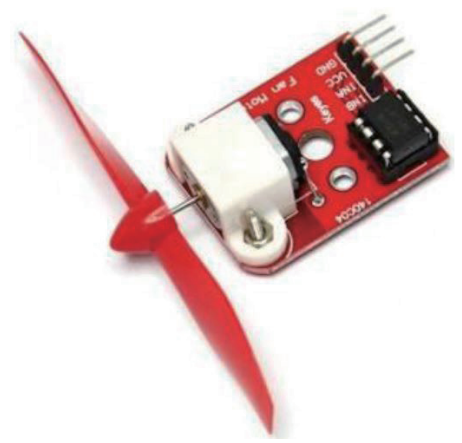

Fig. 6. (Color online) $12 \mathrm{~V}$ direct current fan used in simulation.
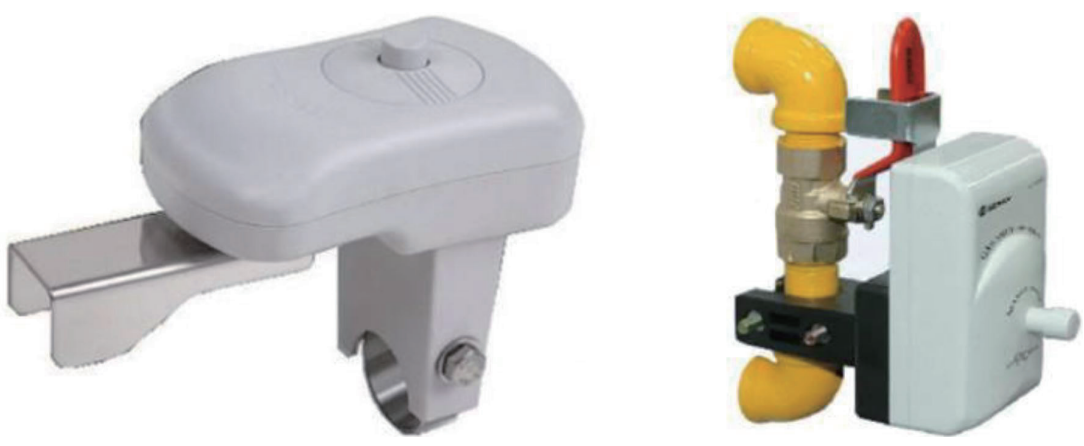

Fig. 7. (Color online) Gas shutoff device.

conditions; an "action" denotes the process that is subsequently implemented; and the entire process is called a "task."

\subsubsection{Line-based notification system}

Because of its application with the Internet and the convenience of Line groups, Line has been widely used in Taiwan and abroad. ${ }^{(28)}$ The line communication platform allows the transmission of texts, images, and videos. Line groups are extensively used as a tool for community exchange, communication, and management in which members can discuss a specific matter with others in the group. This study employed an IFTTT platform to distribute real-time information (Fig. 8) measured by each sensing component in a smart building to Line users or groups.

\subsubsection{Door locks}

Upon detecting an excessively high $\mathrm{CO}$ concentration, the system automatically unlocks the front door (Fig. 9) for the convenience of the community management team or rescuers entering the house for emergency rescue. 


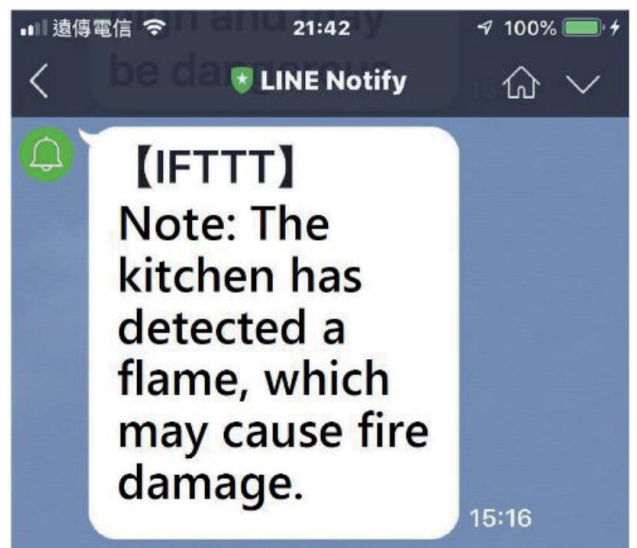

Fig. 8. (Color online) Screenshot of Line notification message.

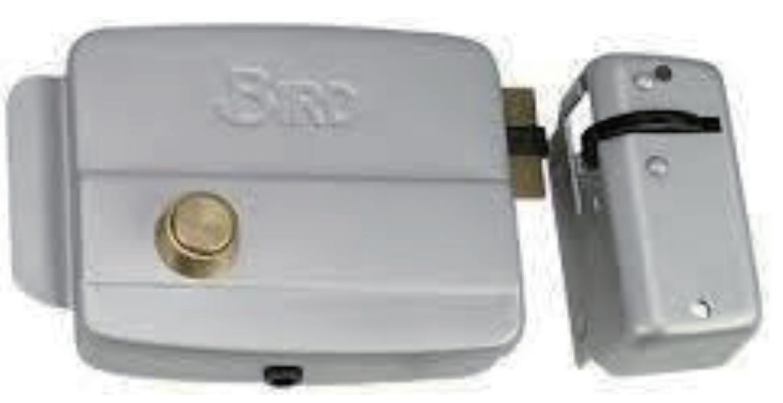

Fig. 9. (Color online) Electric and manual door locks.

\subsubsection{Liquid crystal display module}

This study employed a 16 characters $\times 2$ lines monochrome liquid crystal display module (No. WO1602I; Fig. 10) ${ }^{(29)}$ to display the measured CO concentration in the environment. The name, brand, model, function, and price of the hardware used in this study are listed in Table 2. The total cost of the materials used in this study was US\$341.00.

\section{Results}

The framework of the proposed $\mathrm{CO}$ poisoning prevention system is displayed in Fig. 11. In this study, the Arduino control board is used as the control panel (middle part of Fig. 11). The environmental data is collected by the CO detector (upper part of Fig. 11). The measured value is passed to the Arduino control board and shown on the display. The editor on the right of the figure is the program editing software of Arduino. When the $\mathrm{CO}$ sensor detects a value exceeding the set value, the controllable mechanism at the bottom of the figure can be activated to trigger the alarm, open the window, turn on the fan, open the door lock, send the Line notification message, and turn off the main gas switch. In this study, we developed a $\mathrm{CO}$ poisoning prevention system by combining IoT and information and communication technologies. These three technologies were integrated through the use of relevant sensing components, a controller board, the connection between controlled devices, and the development of management software, thus forming the proposed framework of the prevention system. In addition, the incorporation of software development and notification settings in the Line application enables the proposed system to serve as a reference for the household disaster prevention mechanism.

The MQ-7 CO sensor used in this study can also detect gas. Because creating a scenario with a high $\mathrm{CO}$ concentration is both challenging and harmful to the human body, we used the gas emitted by a lighter to simulate a high $\mathrm{CO}$ concentration, thereby triggering the sensor. 


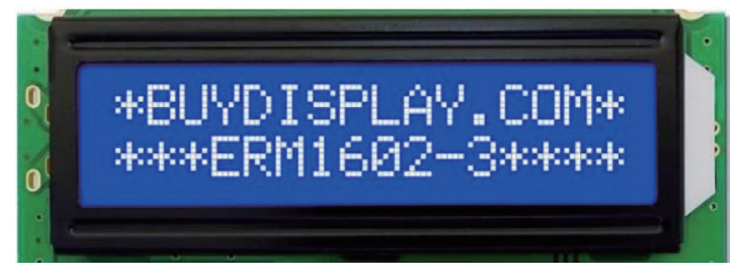

Fig. 10. (Color online) Liquid crystal display module.

Table 2

Hardware used in this study.

\begin{tabular}{lccc}
\hline Name & Brand/Model & Function & Price (US\$) \\
\hline Arduino & Uno & Detects sensor signals and initiates controlled facilities & 6.00 \\
D1 Mini & ESP8266 & Transfers IFTTT and Line information & 3.00 \\
CO detector & MQ-7 & Detects CO intensity & 2.50 \\
Display & Winstar1602/16x2 & Displays detection values of the environment & 3.00 \\
LED alarm & & Generates alert flash to warn residents & 3.50 \\
Audible alarm & K 845755 & Generates alarm sounds to warn residents & 1.00 \\
Relay (3 pieces) & & Commands controllable devices & 3.00 \\
Gas shutoff device & Sunwe/AD-704T & Automatically cuts off, manually recovers & 75.00 \\
Fan & L9110 & Forces air exchange & 5.00 \\
Window opener & \multirow{2}{*}{ Bird } & Opens the window for air exchange & 200.00 \\
Door lock & & Can be automatically opened by the system & 29.00 \\
Miscellaneous hardware & & Wiring and other materials & 10.00 \\
\hline \multicolumn{2}{r}{} & & \\
\hline
\end{tabular}

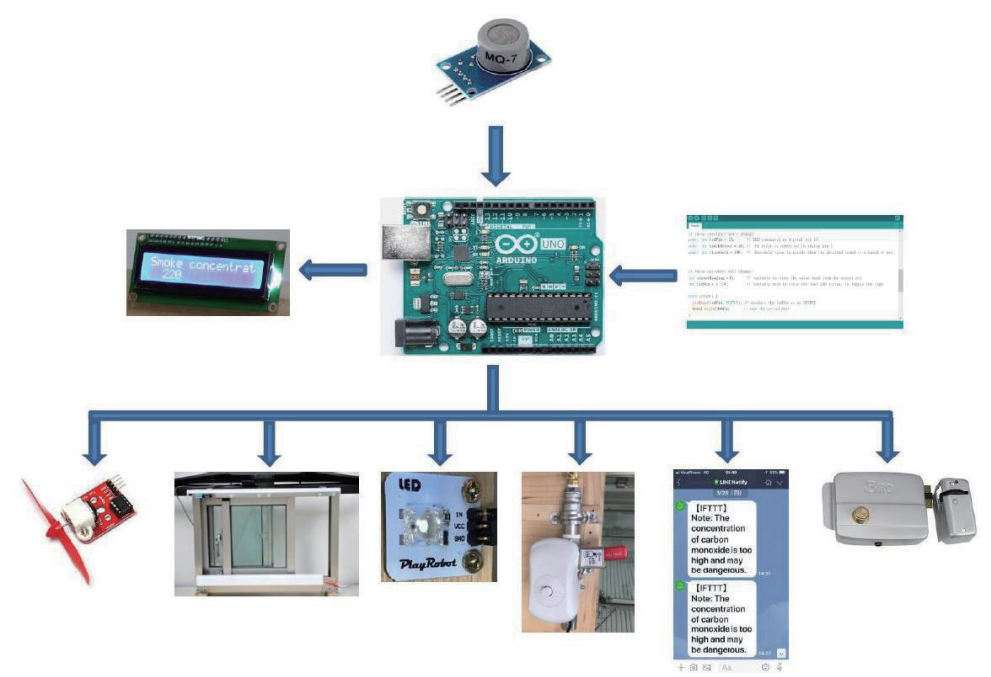

Fig. 11. (Color online) Framework of the proposed CO poisoning prevention system.

After establishing a connection and testing the sensors on the controller board, the changes in $\mathrm{CO}$ concentration triggered by the gas emitted by the lighter appeared on the display module (Fig. 12). When the $\mathrm{CO}$ concentration reached a designated value, the alarm activated the strobe lights and siren (Fig. 13). Then, the fan was turned on (Fig. 14), the windows were opened 
L:artanar raroxide

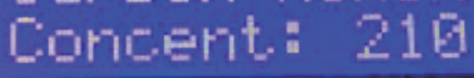

(a)

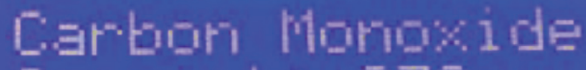

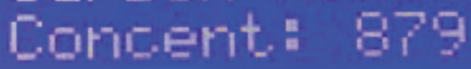

(c)

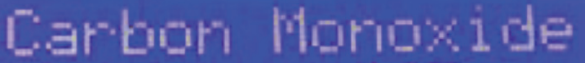 마므로.: 447}

(b)

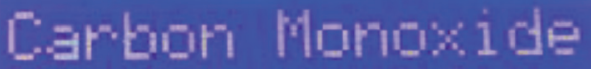 Corotert: 939}

(d)

Fig. 12. (Color online) Changes in CO content. (a) 201, (b) 447, (c) 879, and (d) 939.

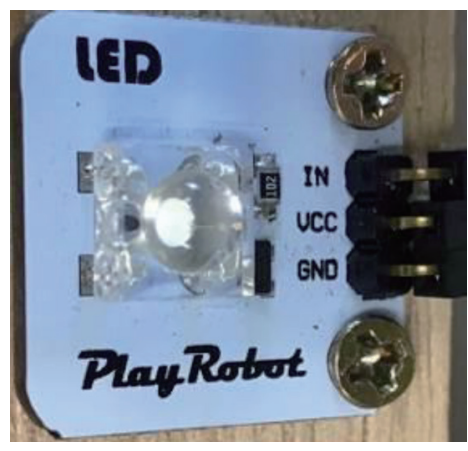

(a)

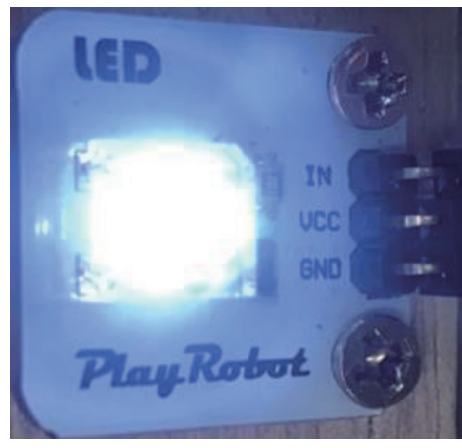

(b)

Fig. 13. (Color online) Activation of the alarm. (a) Alarm before activation and (b) activated alarm.

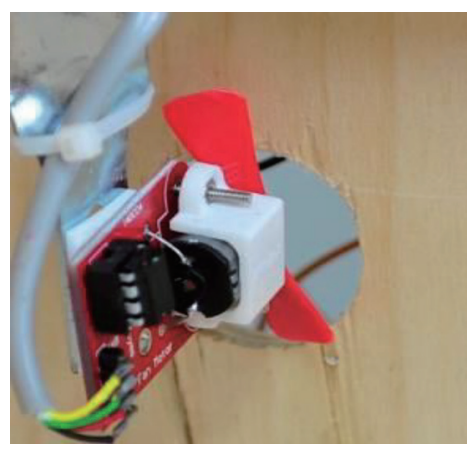

(a)

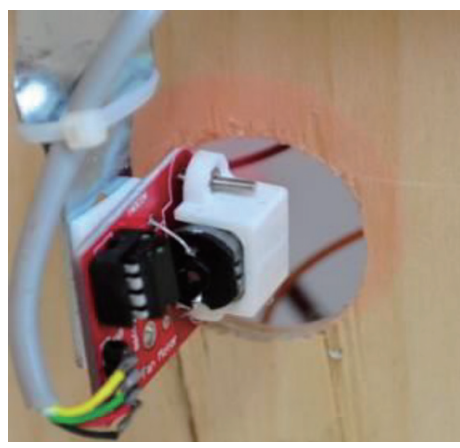

(b)

Fig. 14. (Color online) Activation of the fan. (a) Fan before activation and (b) activated fan.

automatically (Fig. 15), the gas shutoff device was rotated $90^{\circ}$ to cut off the gas supply (Fig. 16), a Line notification popped up on users' mobile phones (Fig. 17), and the front door was unlocked (Fig. 18). When the system reads that the detected $\mathrm{CO}$ value exceeds the safe range, the warning system, the opening of the window, the Line notification, the opening of the door lock, and the shutting off of the gas will be triggered immediately. The automatic gas cut-off device takes about 5-6 s to completely shut off the gas supply. 


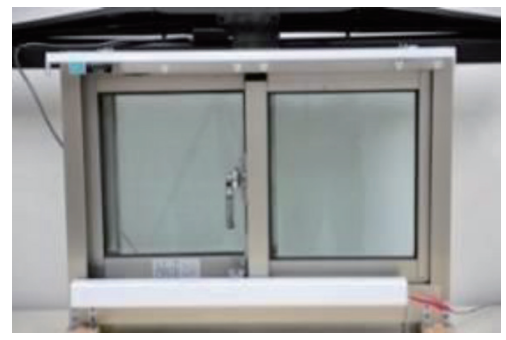

(a)

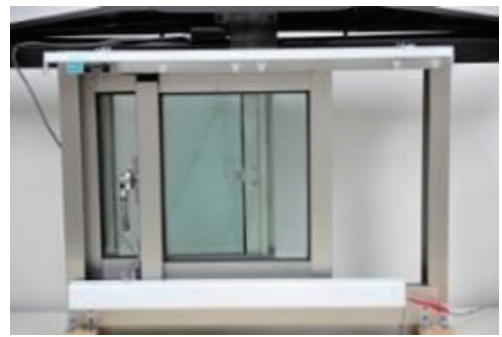

(c)

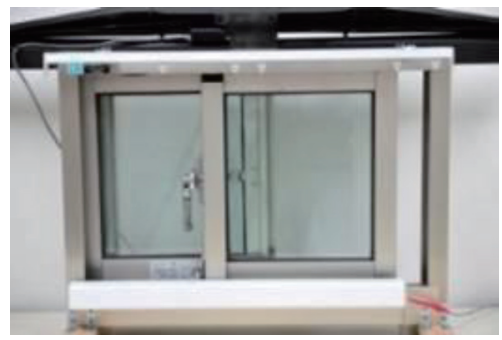

(b)

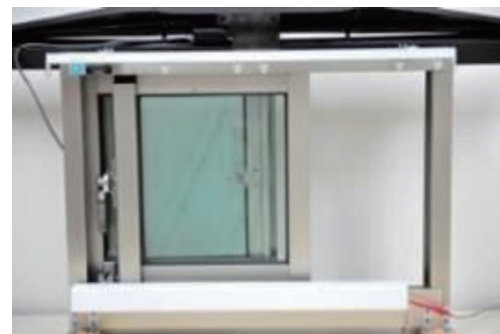

(d)

Fig. 15. (Color online) Activation of the automatic window opener. (a) Closed windows, (b) windows beginning to open, (c) windows almost opened, and (d) opened windows.

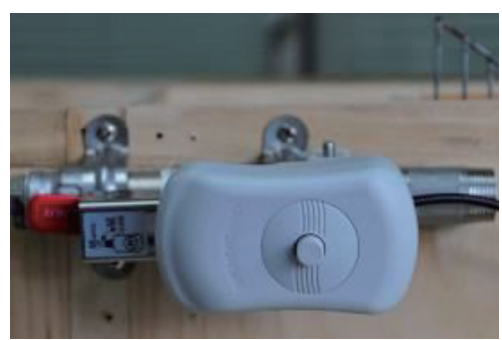

(a)

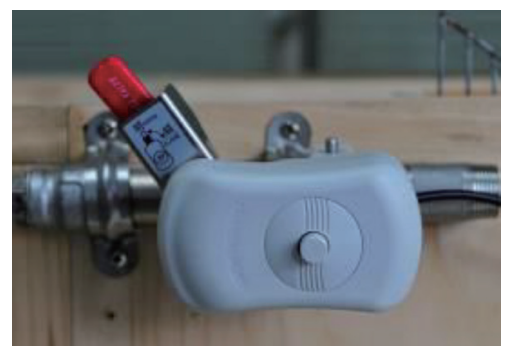

(c)

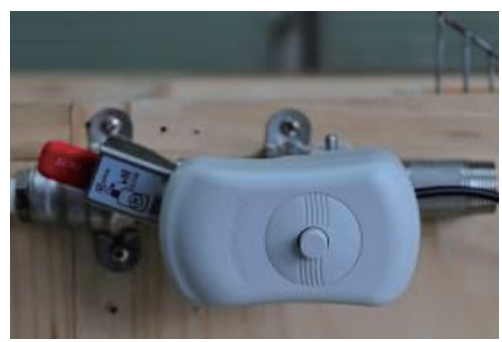

(b)

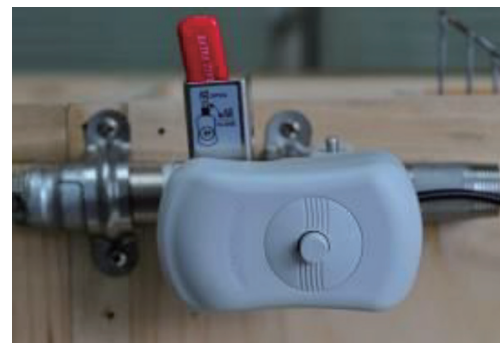

(d)

Fig. 16. (Color online) Gas shutoff device rotating $90^{\circ}$ to cut off gas supply. (a) Gas completely ventilated, (b) shutoff device activated, (c) gas supply being cut off, and (d) gas supply completely cut off. 


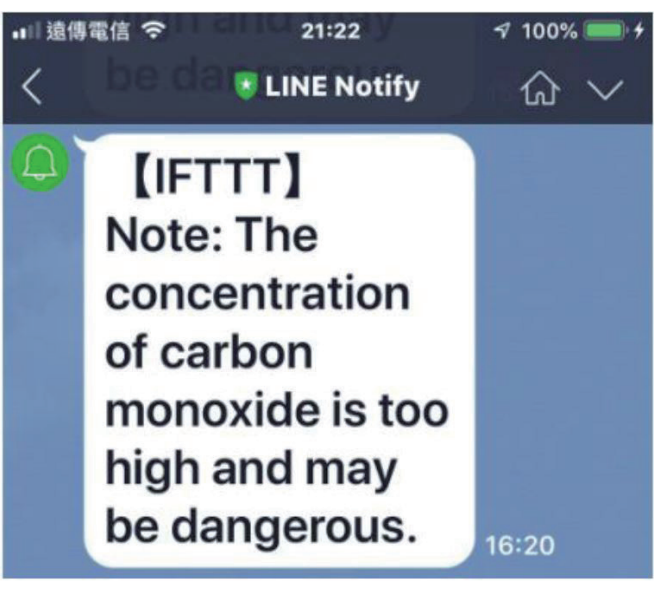

Fig. 17. (Color online) Screenshot of Line notification message on a mobile phone.

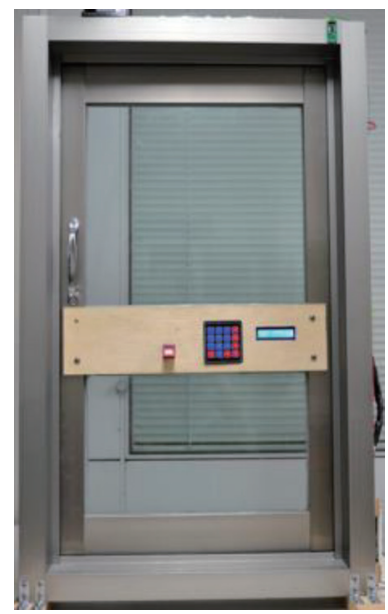

(a)

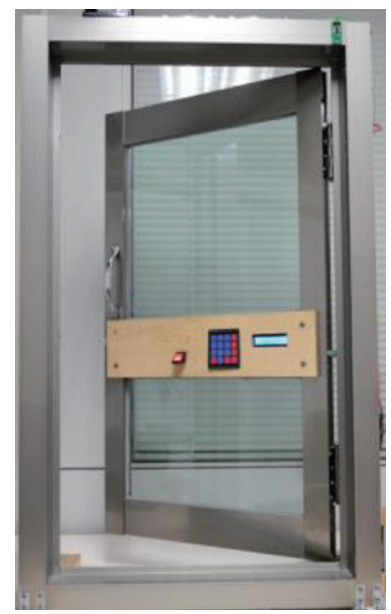

(b)
Fig. 18. (Color online) Front door unlocking. (a) Front door locked and (b) front door unlocked.

\section{Discussion}

The detected values obtained by the MQ-7 CO sensor appear in descending order; a low value implied high $\mathrm{CO}$ content. To simulate the alarm effect of the detected values, the measured values were subtracted from 1000 before being displayed.

At the beginning of this experiment, we employed a normally open electromagnetic valve (Fig. 19) as the gas shutoff device. However, the valve required constant electrical conduction to turn off the gas. Considering that a lack of electrical conduction could enable the continuation of gas emission and thus impose danger, the valve was an inappropriate method for cutting off the gas supply. To completely cut off the gas supply, we used the AD-704T gas shutoff device developed by Sunwe Technology Co., Ltd. When the CO sensor detected a high CO level, the device rotated the switching valve $90^{\circ}$ clockwise to cut off the gas supply, thereby ensuring resident safety.

Compared with the proposed system, patent TW201317946A also had a CO detection module, an alarm device, a gas shutoff device, and a door unlocking mechanism but lacked an automatic window opener, an automatic fan, and a Line-based notification system. Thus, the proposed $\mathrm{CO}$ poisoning prevention system was more complete.

Compared with the proposed system, patent TWM552220U also provided an environment monitoring system in which the environment control device had an automatic window opener and an automatic fan but lacked a Line-based notification system that alerted the community management team and relevant rescue unit to aid in disaster relief in a timely manner.

We are currently displaying the results of the system in our lab. Many faculties, students, and users have visited the lab to experience the design and exchange opinions. Most of them have given positive comments on the system. In addition, our research team has compiled the user opinions as a reference for future study and upgrades recommendations. 


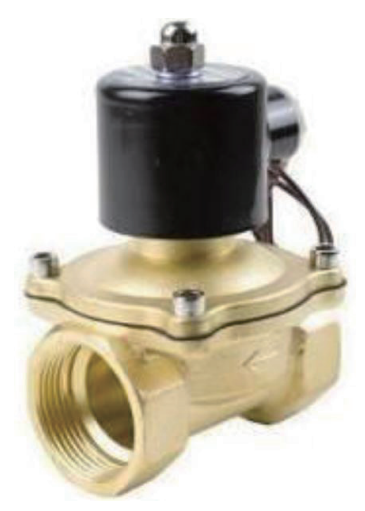

Fig. 19. (Color online) Normally open electromagnetic valve.

\section{Conclusion}

We established a $\mathrm{CO}$ poisoning prevention mechanism capable of performing the following measures upon detecting an excessively high $\mathrm{CO}$ concentration in a house: immediately activating an alarm to warn occupants, cutting off the gas supply, opening the window, turning on a fan for forced ventilation (to reduce the $\mathrm{CO}$ concentration), notifying the residential guard and emergency rescue unit through Line messages, and unlocking the door to allow rescuers to enter for emergency rescue. Such measures can effectively reduce the number of casualties resulting from $\mathrm{CO}$ poisoning in Taiwan every year. Future research directions can be combined with kitchen disaster prevention systems to further increase home safety. Dynamic guiding can be combined with audiovisual technology to provide emergency evacuators with safe guidance about the site environment so that they can quickly escape from the scene.

\section{References}

1 A. L. Llano and T. A. Raffin: Chest 97 (1990) 165. https://doi.org/10.1378/chest.97.1.165

2 National Fire Agency Ministry of the Interior: Carbon Monoxide Awareness Day: Citizen Mobilization for Poisoning Prevention, https://www.nfa.gov.tw/cht/index.php?code=list\&flag=detail\&ids=45\&article_id=540 (accessed April 28, 2019).

3 C.-L. Chen, Y.-Y. Deng, C.-F. Lee, S. Zhu, Y.-J. Chiu, and C.-M. Wu: Sen. Mater. 31 (2019) 1037. https://doi. org/10.18494/SAM.2019.2227

4 C.-C. Chen, G.-N. Sung, W.-C. Chen, C.-T. Kuo, J.-J. Chue, C.-M. Wu, and C.-M. Huang: Sensors 16 (2016) 1568. https://doi:10.3390/s16101568

5 O. Galinina, K. Mikhaylov, S. Andreev, A. Turlikov, and Y. Koucheryavy: EURASIP J. Wireless Commun. Networking 2015 (2015) 178. https://doi.org/10.1186/s13638-015-0393-3

6 H. Ghayvat, S. Mukhopadhyay, X. Gui, and N. Suryadevara: Sensors 15 (2015) 10350. https://doi.org/10.3390/ s150510350

7 C.-C. Huang, C.-H. Ho, Y.-C. Chen, C.-C. Hsu, Y.-F. Wang, H.-J. Lin, J.-J.Wang, and H.-R. Guo: J. Clin. Med. 7 (2018) 349. https://doi:10.3390/jcm7100349

8 Centers for Disease Control Prevention: MMWR. Morbidity and Mortality Weekly Report 54 (2005) 36. https://www.ncbi.nlm.nih.gov/pubmed/15660017

9 L. D. Prockop and R. I. Chichkova: J. Neurol. Sci. 262 (2007) 122. https://doi.org/10.1016/j.jns.2007.06.037

10 National Fire Agency Ministry of the Interior: Promotion for Prevention of Carbon Monoxide Poisoning, https://www.nfa.gov.tw/cht/index.php?code=list\&flag=detail\&ids=984\&article_id=5324 (accessed April 28, 2019). 
11 Medically reviewed by Drugs.com: Carbon Monoxide Poisoning, https://www.drugs.com/health-guide/carbonmonoxide-poisoning.html (accessed April 28, 2019).

12 Fire Bureau Hsinchu County Government: The Five Needs Principle of Preventing Carbon Monoxide Poisoning, http://fire.hsinchu.gov.tw/sense/show.php?itemid=59 (accessed April 28, 2019).

13 P. Spachos and D. Hatzinakos: IEEE Sens. J. 16 (2016) 506. https://doi.org/10.1109/JSEN.2015.2479647

14 S. Abraham and X. Li: Procedia Compu. Sci. 34 (2014) 165. https://doi.org/10.1016/j.procs.2014.07.090

15 V. G. Sanchez, C. F. Pfeiffer, and N.-O. Skeie: J. Sens. Actuator Networks 6 (2017) 11. https://doi.org/10.3390/ jsan6030011

16 M. Chan, D. Estève, C. Escriba, and E. Campo: Comput. Methods Programs Biomed. 91 (2008) 55. https://doi. org/10.1016/j.cmpb.2008.02.001

17 S. Helal, B. Winkler, C. Lee, Y. Kaddoura, L. Ran, C. Giraldo, S. Kuchibhotla, and W. Mann: Proc. First IEEE Int. Conf. Pervasive Computing and Communications (PerCom, 2003) 531. https://doi.org/10.1109/ PERCOM.2003.1192785

18 J. A. Donald: Handbook of Hormones (Academic Press, San Diego, 2016) p. 606. https://doi.org/10.1016/B9780-12-801028-0.00252-X

19 J. A. Raub, M. Mathieu-Nolf, N. B. Hampson, and S. R. Thom: Toxicology 145 (2000) 1.

20 C. F. Lin and Y. H. Wu: Home-Based Multi-Hazard Early Warning System. Publication Number: 201317946, https://twpat-simple.tipo.gov.tw/tipotwoc/tipotwkm?@@1592558688 (accessed April 28, 2019).

21 P. C. Tsai and W. T. Sung: IoT-Enabled Environmental Monitoring System. Publication Number: M552220, https://twpat-simple.tipo.gov.tw/tipotwoc/tipotwkm?.e8410665010010A0000000001^00001000000000000000 00001E62304216 (accessed April 28, 2019).

22 Arduino: What Is Arduino?, https://www.arduino.cc/ (accessed April 28, 2019).

23 TaiwanIOT: MQ-7 Carbon Monoxide Sensor Detection Module, https://www.taiwaniot.com.tw/product/mq-7$\% \mathrm{E} 4 \% \mathrm{~B} 8 \% 80 \% \mathrm{E} 6 \% \mathrm{~B} 0 \% \mathrm{~A} 7 \% \mathrm{E} 5 \% 8 \mathrm{C} \% 96 \% \mathrm{E} 7 \% \mathrm{~A} 2 \% \mathrm{~B} 3 \% \mathrm{E} 6 \% 84 \% 9 \mathrm{~F} \% \mathrm{E} 6 \% \mathrm{~B} 8 \% \mathrm{AC} \% \mathrm{E} 5 \% 99 \% \mathrm{~A} \% \mathrm{E} 6 \%$ A $8 \%$ A1\%E7\%B5\%84-co\%E6\%B0\%A3\%E9\%AB\%94\%E6\%84\%9F\%E6\%B8\%AC\%E5\%99\%A8/ (accessed April 28, 2019).

24 Soon Industrial Co., Ltd.: Electric Sliding Window Opener, https:/goods.ruten.com.tw/item/ show?21538673386091 (accessed April 28, 2019).

25 TaiwanIOT: L9110 Fan Module for Arduino, https://www.taiwaniot.com.tw/product/19110-\%E9\%A2\%A8\%E6 \%89\%87\%E6\%A8\%A1\%E7\%B5\%84-arduino/ (accessed April 28, 2019).

26 Sunwe Technology Co., Ltd.: Gas Shutoff Device, http://www.sunwe.com.tw/gascontrol2.htm (accessed April 28, 2019).

27 IFTTT: Every thing works better together, https://ifttt.com (accessed April 28, 2019).

28 LINE Corporation: Line App Icon Guidline, https://line.me/zh-hant/ (accessed April 28, 2019).

29 Winstar Display Co., Ltd.: Cog Monochrome LCD Display Module 16x2 Model No. WO1602I, https://www. winstar.com.tw/zh-tw/products/cog-lcd-module-display/monochrome-led.html (accessed April 28, 2019).

\section{About the Authors}

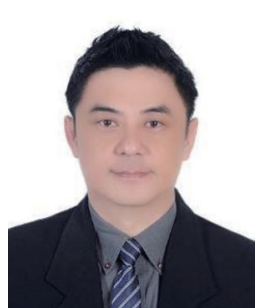

Wei-Ling Hsu received his Ph.D. in 2014 from the Department of Civil Engineering and his Master's degree in architecture in 2009 from Chung Hua University in Taiwan. From 2009 to 2017, he was an adjunct assistant professor at Chung Hua University. He is currently an associate professor at the School of Urban and Environmental Science, Huaiyin Normal University, China. His research expertise includes the application of satellites and unmanned aerial vehicles in ground-level environmental monitoring, urban traffic, urban and rural planning, and the incorporation of data analysis in fuzzy multiple criteria decision making for cities and environments. 


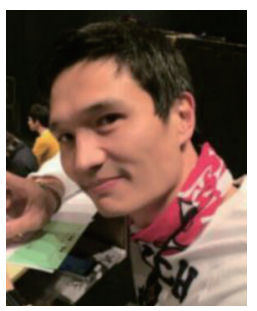

Chih-Yuan Ho received his B.S. and M.S. degrees from Chung Hua University, Taiwan, in 1997 and 2005, respectively. Since 2002, he has worked in a construction engineering consultancy company. Since 2011, he has also been an adjunct lecturer at China University. His research interests include landscape planning and architectural space planning.

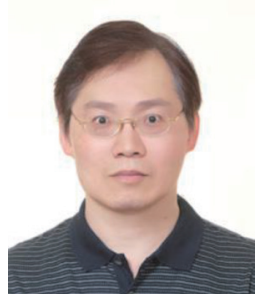

Chiu-Kuo Liang received his Ph.D. degree in computer science from the National Tsing Hua University, Taiwan, in 1990. He is currently an associate professor of the Department of Computer Science and Information Engineering at Chung Hua University. His research interests include wireless mobile computing, sensor networks, RFID systems, and parallel processing.

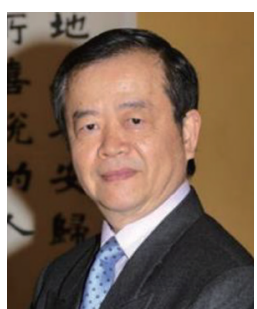

Yan-Chyuan Shiau received his B.S. degree from National Taiwan Institute of Technology, Taiwan, in 1980 and his M.S. and Ph.D. degrees from Texas A\&M University, U.S.A., in 1988 and 1992, respectively. From 2000 to 2012, he was an associate professor at Chung Hua University, Taiwan. Since 2013, he was a professor at Chung Hua University, Taiwan. His research interests are in ICT, IOT and smart home.

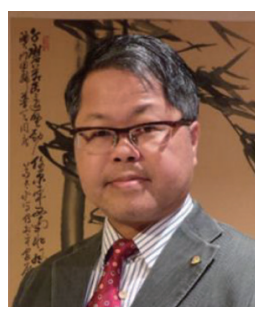

Hung-Nien Hsieh received his M.S degree from National Cheng Kung University, Taiwan, in 1990 and his Ph.D. degree from Tongji University, China, in 2008, respectively. Since 2015, he has been a professor at Chung Hua University, Taiwan. His research interests are in urban planning, industrial environment planning, and intelligent city.

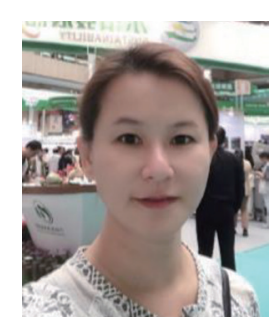

Shu-Chen Lai received her B.S. degree from National United University in 1996 and her M.S. degree from Chung Hua University, Taiwan, in 2014, respectively. She is currently the head of Liangzheng Environmental Technology Co., Ltd. Her research interests include the application of artificial intelligence and Internet of Things in elderly care. 